DOI:

10.1038/nrmicro1415

\section{URLs}

Lactococcus lactis

http://www.ncbi.nlm.nih.gov/ entrez/query.fcgi?db=genome $\operatorname{prj} \delta \mathrm{cmd}=$ Retrieve $\& d o p t=O v e r$ viewElist_uids=12306

Listeria monocytogenes http://www.ncbi.nlm.nih.gov/ entrez/query.fcgi?db=genome prjEcmd=RetrieveEdopt=Ove viewElist_uids=12309

Clostridium botulinum http://www.ncbi.nlm.nih.gov/ entrez/query.fcgi?db=genome prjधcmd=Retrieve\&dopt=Over viewElist_uids=193

NisC

http://ca.expasy.org/uniprot/ Q03202

NisA

http://ca.expasy.org/uniprot/ P13068

NisP

http://ca.expasy.org/uniprot/ Q07596

ANTIMICROBIALS

\title{
A ringing success
}

In a recent issue of Science, Satish Nair, Wilfred van der Donk and colleagues report on an important advance in the understanding of nisin biosynthesis - a finding that could help the search for new antibiotics.

Nisin is a post-translationally modified lantibiotic that is naturally produced by Lactococcus lactis and is effective against a wide range of Gram-positive bacteria, including food-borne pathogens such as Listeria monocytogenes and Clostridium botulinum. It is therefore widely used in the food industry as a preservative. Nisin's microbicidal properties work through several mechanisms: by binding to lipid II - an essential intermediate in peptidoglycan biosynthesis - nisin induces pore formation in the membrane and disrupts cell-wall biosynthesis; in addition, nisin inhibits the outgrowth of bacterial spores.

Nisin contains five thioether rings of varying sizes and conformations that are required for its biological activity. Li et al. have now reconstituted the cyclization process of nisin in vitro, and report that the cyclase $\mathrm{NisC}$ is responsible for the formation of all of the thioether rings. NisA, the precursor peptide, is ribosomally synthesized, followed by dehydration of serine and threonine residues. $\mathrm{Nis} C$ then catalyses five distinct cyclization reactions to generate five cyclic thioethers: one lanthionine and four methyllanthionines. Once cyclization is complete, NisP removes the leader peptide. Leader-peptide recognition probably has a role in NisC binding, as NisC was unable to convert the dehydrated peptide into its biologically active form after cleavage of the leader peptide.

Li et al. also determined the X-ray crystal structure of $\mathrm{NisC}$, which revealed unexpected similarities in fold and substrate activation with mammalian farnesyl transferases. Similar to farnesyl transferase, a single zinc ion is situated at the top face of the double $\alpha$-barrel in NisC. These structural similarities suggest that human homologues of NisC might post-translationally modify a cysteine of a protein substrate.

Although nisin has been in use as a food preservative worldwide for decades, it has not induced the widespread biological resistance encountered by many antibiotics. This is thought to be due to its multiple modes of action and the nature of the lipid II target, which is not a protein and is not easy to modify by the bacteria. The newfound knowledge of how nisin is biosynthesized, and the enzymes involved in this process, could be useful in the search for novel antibiotics that could avoid the development of antibiotic-resistant bacteria.

Annie Tremp
ORIGINAL RESEARCH PAPER $L i$, B et al. Structure and mechanism of the lantibiotic cyclase involved in nisin biosynthesis. Science 311, 1463-1467 (2006)

FURTHER READING Christianson, D. W. Five golden rings. Science 311, 1383 (2006) | Cotter, P. D., Hill, C. \& Ross, R. P. Bacteriocins: developing innate immunity for food. Nature Rev. Microbiol. 3 777-788 (2005)

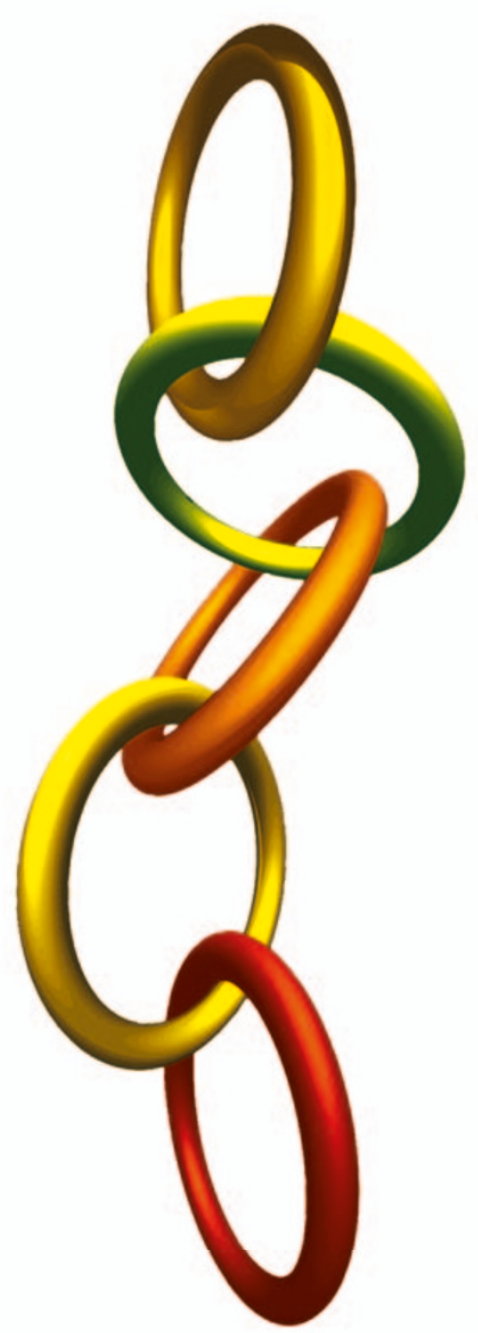

